\title{
Space, time, parallelism and noise requirements for reliable quantum computing
}

\author{
A. M. Steane \\ Department of Physics, Clarendon Laboratory, Parks Road, Oxford, OX1 3PU, England
}

(July 1997)

\begin{abstract}
Quantum error correction methods use processing power to combat noise. The noise level which can be tolerated in a fault-tolerant method is therefore a function of the computational resources available, especially the size of computer and degree of parallelism. I present an analysis of error correction with block codes, made fault-tolerant through the use of prepared ancilla blocks. The preparation and verification of the ancillas is described in detail. It is shown that the ancillas need only be verified against a small set of errors. This, combined with previously known advantages, makes this 'ancilla factory' the best method to apply error correction, whether in concatenated or block coding. I then consider the resources required to achieve $2 \times 10^{10}$ computational steps reliably in a computer of 2150 logical qubits, finding that the simplest $[[n, 1, d]]$ block codes can tolerate more noise with smaller overheads than the $7^{L}$-bit concatenated code. The scaling is such that block codes remain the better choice for all computations one is likely to contemplate.
\end{abstract}

Large-scale quantum computation can be regarded either as a realistic future possibility, or as a theoretical device which enables basic questions to be asked about quantum mechanics and the computational power of machines. In either case, an important fundamental issue is that of the conditions required to enable a given computational problem to be solved on a quantum computer. Until recently, it was believed that the effects of noise were more or less insurmountable in a complex quantum system such as a quantum computer. However, two discoveries have greatly shaken this belief. These are the possibility of efficient quantum error correction for arbitrarily large computers [1]2], and the possibility of faulttolerant correction and computing 3,4 ,

An important third idea is that of concatenated coding, [5.6. The use of concatenated coding, combined with fault-tolerant methods, enables the important threshold result to be obtained 6 11]. This result states that in order for arbitrarily long computations to be possible, the noise level $\gamma$ per elementary operation need not be arbitrarily small, but need only be below a threshold value $\gamma_{0}$. The noise must have various special properties for this result to be valid, such as that the effects of noise at different qubits and at different times are independent, that $\gamma$ does not grow when the computer is made larger, and that arbitrarily many elementary operations can be performed in parallel.

The threshold result is an important element of the basic theory of the control of noise in a quantum com- puter. However, just as it is meaningless to talk about the amount of noise without stating what type of noise one is dealing with, it is also meaningless to talk about a threshold error rate without stating what other computational resources are needed. For example, it is easy to see that the greater degree of parallelism one assumes, the less stringent are the requirements on memory errors in a quantum computer. The threshold result is really a statement about how the noise requirements scale with other requirements. The inevitable cost of the threshold is that concatenated coding is inefficient in both space (number of qubits) and time (number of elementary operations), compared with non-concatenated codes. In view of this, it is important to compare different coding and correction methods on the basis of all the relevant parameters.

Fault-tolerant error correction using non-concatenated codes is very powerful if we take advantage of the approach I put forward in an earlier paper [12]. In this approach, the task of error correction is reduced essentially to one of quantum state synthesis. The most important task for a quantum computer is to generate verified copies of the encoded zero state $\left|0_{E}\right\rangle$ in ancilliary blocks of qubits. I will refer to this method as the 'ancilla factory' method.

For sufficiently long computations, a concatenated correction method is probably the best choice because of the threshold result. However, the estimates in [12 suggest that the size of computation (number of logical qubits times number of computation steps) may well be very large $\left(>10^{15}\right)$ before concatenated coding is preferable to simple block coding with the ancilla factory method. The present paper will elucidate this by providing a more complete analysis of the ancilla factory than was done previously. In particular, the network required to verify the ancilla state is fully specified and taken into account. It is noteworthy that this network performs a minimal error detection, in a sense to be described. Also, the usefulness of measuring the syndrome and using classical processing to interpret it, rather than a further quantum network, will be underlined. I will show that the ancilla factory method makes error correction using CSS (Calderbank, Shor and Steane [1,2], see also [16]) codes sufficiently efficient that it compares favourably with the concatenation method, even for a large computational task such as factorisation of a 130 digit (430 bit) number using Shor's algorithm.

The main conclusion of this study is a 'bench mark' indication of the power of different error correction methods, in terms of four parameters: the noise level, the 'scale up' (increased number of qubits), the 'slow down' 
(increased number of operations) and the degree of parallelism (ability to apply many gates simultaneously) required to make a computer sufficiently reliable to carry out a specific difficult task. This bench mark is offered as a target for others to bring down, either by optimisation of current methods or by finding radically new ones. It is a rough guide to the hardware which would be needed if large scale quantum computations were to be attempted one day.

\section{CHOICE OF METHOD}

There are two requirements for error correction techniques to be used to stabilize a quantum computer. These are fault-tolerant error correction, and fault-tolerant computational operations. Two approaches to faulttolerant error correction were put forward by Shor [3] and Kitaev [₫. In Shor's approach, the quantum error correcting code (QECC) is taken from a subset of the CSS codes described in [1.2], and the syndrome extraction is carefully arranged so as to restrict the propagation of errors. In Kitaev's approach the QECC is itself designed so that each qubit is involved in a bounded number of check operators (a 'local check code'), so that even a straightforward extraction of the syndrome will not propagate errors too far. Shor's fault-tolerant syndrome extraction was generalised to all stabilizer codes [13,14 by DiVincenzo and Shor 115. The ancilla factory method which I have proposed is closely related to Shor's ideas. Since it is to my knowledge the best method currently known, it is used in this study.

Aharanov and Ben-Or [8] have discussed fault-tolerant methods in which syndromes are not measured (ie converted into classical information) during the computation, but are interpreted within the unitary quantum evolution. The advantage of avoiding measurements is that the analysis is simpler, and measurements will almost certainly be slow compared to unitary operations in an experimental system. However, the disadvantage is that the quantum computer must be made much larger in order to store the extra 'garbage' manufactured by error corrections, and measurements provide robust classical information which is very useful since much of the syndrome interpretation can then be done classically. For these reasons I retain the use of measurements.

Fault-tolerant computational operations have been discussed for various types of stabilizer code. A universal set of operations was described by Shor [3] for CSS codes obtained by puncturing a self-dual classical code whose words all have weight equal to a multiple of four ('doubly even' codes). Further methods were described by Knill et. al. [7] for codes obtained by puncturing one with with weights divisible by 8 and dual distance $\geq 4$. The same authors give some more techniques in [10], based partly on the ideas of Kitaev [6]. Rotations, such as those needed in a Fourier transform network, are considered by
Preskill [11]. Gottesman has described methods by which a universal set of operations can be obtained for any stabilizer code [16], though some of these methods are considerably more complicated than the ones designed for particular codes.

The general rule is that the more symmetry and simplicity there is in the code construction, the simpler are the fault-tolerant methods. This study will be restricted to quite simple codes, namely $[[n, 1, d]]$ CSS codes obtained by puncturing a doubly even classical self-dual code. The notation $[[n, k, d]]$ refers to a QECC encoding $k$ qubits into $n$, with minimum distance $d$, thus $t=(d-1) / 2$-error correcting. Note that we choose to encode only a single qubit in each block, and restrict ourselves to doubly-even codes in order to allow a large set of bitwise operations. It may well be worth while to sacrifice some of the simplicity in the computational operations in order to gain more efficient storage; this is an avenue for future work.

\section{ASSUMPTIONS}

In order to estimate the reliability of the encoded quantum computer, we will make a set of standard assumptions about the noise, which have been discussed by several authors [5, 10,11]. Note that much more general error models have been considered by Knill et. al. [17, 1, 10. The noise model assumes independent stochastic errors among the qubits, whose overall effect can be estimated by adding classical error probabilities rather than quantum amplitudes. In other words, to keep the problem managable we will only try to find out the limitations imposed by such random errors.

We will adopt the depolarising channel model for the noise on each qubit [18]. For a given error opportunity, a qubit remains unchanged with probability $1-p$, and undergoes $\sigma_{x}, \sigma_{y}$ or $\sigma_{z}$ errors with equal probabilties $p / 3$. Of all the errors that occur, on average $2 / 3$ will require correction in the computational basis $\{|0\rangle,|1\rangle\}$ and $2 / 3$ in the conjugate basis $\{|0\rangle+|1\rangle,|0\rangle-|1\rangle\}$. The errors $\sigma_{x}$ and $\sigma_{z}$ will be referred to as 'bit errors' and 'sign errors' respectively.

To model the noise in a gate operating on two qubits, we assume the gate produces the action it should, followed by error operators. There are sixteen possible error operators, all the members of the set $\left\{I, \sigma_{x}, \sigma_{y}, \sigma_{z}\right\}$ $\otimes\left\{I, \sigma_{x}, \sigma_{y}, \sigma_{z}\right\}$. If the gate error probability is $p$, we assume the error-free case $I \otimes I$ occurs with probability $1-p$, and the other 15 cases occur with equal probabilities $p / 15$. However, we will not take advantage of the fact that errors in the two qubits are correlated (for example if a bit-error is found in the first qubit, the probability of bit-error in the second qubit rises from $(8 / 15) p \ll 1$ to $1 / 2$.) Rather, we assume that with probability $O(p)$ (not $p^{2}$ ) errors are produced in both qubits, but we must correct the errors as if they were independent. Thus we 
over-estimate the difficulty of error correction. Furthermore, we will overestimate the probability $8 / 15$ as $2 / 3$, to simplify the equations.

As usual in quantum error correction, when we talk of errors occuring with given probabilities, we refer to the probabilities that the quantum state is projected onto each state of well-defined error syndrome, when the syndrome is later extracted. We allow for relaxation in the system by permitting states of different syndrome to be entangled with different environment states [2,17].

The most important, and possibly unphysical, assumption is that error probabilities can be multiplied. That is, if one error has probability $p_{1}$, and another $p_{2}$, the probability of getting both is $p_{1} p_{2}$. This is reasonable if errors at different places and times are uncorrelated with one another, and it corresponds to the standard Gaussian noise model commonly used in physics. However, noise in real systems often has two components: one which can be well described in terms of standard Gaussian statistics, where the probability of obtaining an event many standard deviations from the mean falls exponentially with the distance from the mean, and another component in which the wings of the probability distribution are much higher. In other words, rare events are much more likely in practice than one would suppose on the basis of the standard treatment of experimental error. The correction methods discussed here cannot provide stability against such noise. In order to handle it, others methods must be used in conjuction with the error correction we are considering.

The overhead required to implement error correction depends on the noise level. When estimating the scaling of this overhead with the noise level, it is convenient to assume that the noise level per qubit is independent of the total number of qubits in the computer. Such an assumption is required, for example, for the threshold result for concatenated codes. This is unlikely to be valid for a real device. However, when giving the noise level required for a task on a computer with a specified number of qubits, no assumption about the scaling of noise need be made.

Another consideration is that of parallelism, ie the number of quantum gates that can be executed in parallel in a single time step. A large degree of parallelism turns out to be necessary in order to keep storage errors under control. For this reason we will be explicit about the degree of parallelism involved in the methods to be discussed.

Most of the quantum gates in the stabilized computer will be involved in error correction rather than the execution of a computational step. For this reason most two-qubit gates only connect qubits within a few blocks of one another. However, a computational step might involve pairs of qubits anywhere in the computer. It will be assumed that such gates can be applied to any pair of qubits without any significant extra cost.

Fault tolerant quantum computation makes much use of state preparation and measurement. These irreversible operations involve an amplification of some kind if they are to be done reliably. They are therefore much slower than a unitary quantum gate. In a practical system one would probably wish to allow many gates to be carried out on other (unrelated) qubits during the time any given qubit is prepared or measured, but this will complicate the analysis here. We will allow the same time (in fact one time step) for a gate as for a measurement. Therefore the results are strictly valid only if the time step is taken to be the time necessary for a measurement rather than a gate. However, there are many more gates than measurement operations in the networks actually used, therefore it is a fair approximation to take the time step to be that of a gate.

A further question of speed of operation is that involved in decoding of the error syndromes. This is done by a dedicated classical computer, and we assume that the result is available within the time required to prepare an ancilla state in the quantum computer. This is itself quite a large classical computation, involving decoding thousands of $(n-1)$-bit syndromes, where $n=23,55,87$ for the codes to be discussed.

Finally, the important question of leakage errors is here assumed to be solved, for example by converting leaked qubits into erroneous ones, using leak detection circuits as in 11]. The overhead represented by such additional circuits will not be included in the analysis.

\section{ANALYSIS}

We will adopt the following notation. The computation to be performed requires $K$ logical qubits and $Q$ computational steps. This is achieved in a set of $N>K$ physical qubits using $T>Q$ elementary gates. $N$ is of order $n K$, where $n$ is the block size of the $[[n, 1, d]]$ CSS code used. The number of correctable qubit errors per block is $t=(d-1) / 2$. The parameters $K, Q, N, T, n, t$ are integers. The noise is parametrised by two real numbers, $\gamma$ the gate or measurement error probability, and $\epsilon$ the store error probability per qubit per time step.

The parameters of the logical computer are $K$ and $Q$. This logical computer is embedded in a physical computer of parameters $N$ and $T$. We use the language 'computational step' to mean a gate on encoded qubits which evolves the logical computation, and 'elementary gate' for a gate on bare qubits. The main overheads involved in error correction are the 'scale-up' $N / K$ and the 'slowdown' $T / Q$ (number of gates per computational step). The amount of time required to finish the computation is of order $T$ divided by the degree of parallelism.

\footnotetext{
${ }^{1}$ Roughly speaking, this is an amplification from the quantum to the classical level.
} 
We will analyse a complete fault-tolerant computation, using correction by a block code, and then compare with the requirements for a concatenated code, drawing on the work of Preskill [11].

The 'ancilla factory' error correction method is based on the generation of the encoded zero state $\left|0_{E}\right\rangle$, as described in 12 . The $n$-qubit block to be corrected will be referred to as $b$, and the $n$-qubit ancilla as $a_{x}$. A further qubit is used for verification of $a_{x}$. We imagine that error correction is carried out in parallel on all the $K$ blocks in the computer. However, within a block we will restrict parallel operation to single-bit gates 2 . The state $\left|0_{E}\right\rangle$ is generated in $a_{x}$ and then rotated by the Hadamard transform $H$ applied bitwise. Since we are using [[n, 1, $d]]$ CSS codes this produces the state $\left|0_{E}\right\rangle+\left|1_{E}\right\rangle$ (ignoring normalisation). The network to perform these operations is given in fig. 1 for the 7 -bit code $[[7,1,3]]$ [19. The general features of the method were described in [12], but here we will be specific about the networks and the propagation of errors. The network in fig. 1 is designed both to generate and to verify the state required. If an error is detected, the network is restarted from the beginning. The verification only picks up bit errors (Pauli $\sigma_{x}$ operator) in the state, not sign errors (Pauli $\sigma_{z}$ operator). This is because bit errors would be converted into sign errors by the Hadamard transform, and then carried into $b$ by the subsequent XoR operations, so we must guard against them. Sign errors will be converted into bit errors by the Hadamard transform, and result in an incorrect syndrome. The fact that sign errors (before the $H$ gates) can propagate from one qubit to many is not a problem since in any case we will assume that a single sign error makes the syndrome invalid. A bit error, before the verification gates, can also propagate from one qubit to many, but such errors will be detected and the ancilla preparation restarted. The remaining undetected bit errors are uncorrelated.

Meanwhile a separate ancilla $a_{z}$ is prepared, in parallel (see fig. 3), to be used for correction of sign errors in $b$. The preparation network is the equivalent of fig. 1 but in the conjugate basis.

Let us first confirm the claim about the verification of the ancilla, namely that only uncorrelated sign (bit) errors are finally coupled from $a_{x}\left(a_{z}\right)$ into $b$. The network of fig. 1 can be divided into three parts: generation, verification, and Hadamard transformation. First assume the verifier gates are noise-free. Then the most general state which can survive the verification is

$$
\sum_{i \in C} c_{i}|i\rangle \otimes\left|e_{i}\right\rangle
$$

where $C$ is the classical $[7,3,4]$ code and $\left|e_{i}\right\rangle$ is a state of the environment. In order to analyse the coupling to $b$ we

\footnotetext{
${ }^{2}$ If this assumption is relaxed, as for example in 11, the restriction on memory error rate is less severe.
}

make use of the simple equivalence shown in fig. 2. The most general error-free state of $b$ is $\alpha\left|0_{E}\right\rangle+\beta\left|1_{E}\right\rangle$. The Hadamard transformation of $b$ (see fig. 2) carries this state to $\sum_{j \in C}(\alpha+\beta)|j\rangle+(\alpha-\beta)|\neg j\rangle$, written using the same basis states as eq. (11). To obtain this form we have made use of the CSS code construction $C \in C^{\perp}$. The XOR between $a_{x}$ and $b$ produces

$$
\begin{aligned}
& \sum_{i \in C} c_{i}|i\rangle\left|e_{i}\right\rangle \sum_{j \in C}(\alpha+\beta)|j+i\rangle+(\alpha-\beta)|\neg j+i\rangle \\
= & \sum_{i \in C} c_{i}|i\rangle\left|e_{i}\right\rangle \sum_{j \in C}(\alpha+\beta)|j\rangle+(\alpha-\beta)|\neg j\rangle
\end{aligned}
$$

since displacing a linear code by a member of the code simply moves the code onto itself. Hence the most general noisy state of $a_{x}$ which survives noise-free verification produces no errors in $b$. The significant point is that this is achieved with only a weak verification: one which detects none of the sign errors and only those bit-error vectors which are not in $C$, which is a small set (only $2^{4}$ out of $2^{7}$ ).

When we allow the verification to be itself noisy, its gates introduce both bit and sign errors. However, the verification network is one in which bit errors can not propagate among the qubits of $a_{x}$, therefore the bit errors which are produced are uncorrelated. The same is true for the Hadamard transformation of $a_{x}$ at the end of fig. 1 .

The network of fig. 1 involves 46 elementary operations and 30 time steps. An elementary operation is taken to be a gate applied to bare (non encoded) qubits, or a preparation or measurement of a bare qubit. It is assumed that single-qubit elementary operations can be carried out in parallel with double-qubit ones. More generally, if the generator matrix of the classical code giving $\left|0_{E}\right\rangle$ in the quantum code has $m$ rows, and the average weight of a row is $w$, then the numbers of operations and time steps are as follows.

There are $n+1$ preparation operations, $m+1$ measurement operations, and $n+m$ Hadamard gates, making $2(n+m+1)$ single-bit operations. There are $m(w-1)$ XOR gates between qubits in $a_{x}$, and $m(w+1)$ XOR gates between $a_{x}$ and the verification bit, where we have used the fact that the code is $[[n, 1, d]]$, so the final parity check in the verifier needs $m$ gates (this verifies that $\left|0_{E}\right\rangle$, not $\left|1_{E}\right\rangle$, has been generated). The number of time steps is

$$
m(w-1)+2+m(w+1)+m+1=m(2 w+1)+3 .
$$

For the $[[n, 1, d]]$ CSS codes which we are considering, $m=(n-1) / 2$ and $w=d+1$ [2]. The number of time steps is thus $n w+(n+5) / 2-w$. This completes the preparation of $a_{x}$.

Once the ancilla state is prepared, $a_{x}$ and $b$ interact by an XOR gate applied transversally, ie $n$ elementary operations, and then the ancilla is measured. These xor gates 
are applied sequentially, not in parallel. The syndrome is extracted from the measurement results. Let $1-\alpha$ be the probability that the correct syndrome is obtained. This requires no bit errors to be present in $a_{x}$ when the syndrome is extracted, whether from gates or free evolution, so

$$
1-\alpha \simeq\left(1-\frac{2 \gamma}{3}\right)^{2(m w+2 n+m+1)}\left(1-\frac{\epsilon}{3}\right)^{(n-1)(m(2 w+1)+3)} .
$$

In writing this expression, we have taken into account the fact that most $\sigma_{y}$ errors produced in $a_{x}$ before the final verification will be detected, so we only need account for $\sigma_{z}$ memory errors, of probability $\epsilon / 3$. This is also why the error probability associated with the XOR gates is accounted $2 \gamma / 3$ rather than somewhat larger. Two of the timesteps (initial preparation, and the Hadamard transform) do not involve any free evolution. Apart from this, we have neglected the slight reduction in storage errors obtained by preparing qubits just before they are needed. We will find later that we require $(\alpha / n)^{t+1} \ll 1 / n K Q$, so $\alpha$ is small (eg $\sim 0.001$ ) and most of the time the ancilla preparation is succesful. The ancilla verification plays an important role in rejecting failures, but these are rare occurences.

The ancilla preparation and syndrome extraction is repeated at least $r$ times before any attempt is made to correct the block $b$. An error may well be generated in $b$ during this process, in which case the syndrome should change. Therefore in making the best guess of what corrective measure to apply to $b$ on the basis of the syndrome information obtained, allowance must be made for such possibilities. In this analysis I will assume that on average $r=t+1=(d+1) / 2$ syndromes are needed. The logic of this choice will become apparent shortly. Usually the first $r$ syndromes will be consistent (probability $\left.\sim(1-\alpha)^{r}>0.99\right)$. Either they agree, or they are consistent with an identifiable error developing during the free evolution of $b$. When they are not consistent, usually just one more syndrome will suffice to produce an unambiguous interpretation, though we allow ourselves to generate $O(r)$ more syndromes, before correcting, on the rare occasions that this is necessary.

The probability of getting a set of $r$ syndromes consistent with one another but all incorrect, and thus of applying the wrong 'corrective' measure to $b$, can be estimated as the probability that $r$ successive ancillas all develop an error in the same qubit?. This is of order $2 n(\alpha / n)^{r}$. We consider the whole computation to fail if this happens. We will find shortly that a much more likely cause of failure is that too many errors accumulate

\footnotetext{
${ }^{3}$ This part of the analysis differs from 12] which was overly cautious at this point.
}

in $b$, so even if we have greatly underestimated the probability of using the wrong syndrome, the final conclusions about error rates will be unaffected. The choice $r=t+1$ is perhaps an 'overkill', but it is the logical choice in order to ensure that the syndrome failure probability scales with $t$ in the same way as the probability of generating too many errors in $b$.

We now wish to find how many errors accumulate in $b$ during the course of the computational steps, the ancilla preparations, and the interaction between $a$ and $b$. It is very important to allow for errors in the free evolution of the qubits (storage errors) as well as gate errors, since the qubits spend much more time 'resting' than in gate operations.

Most of the operations on any block $b$ come from the error corrections rather than the computational steps. This is because most computational steps involve operations on only a few of the $K$ logical bits. The remaining $K-2$ or so blocks freely evolve and then require correction for storage errors, and for the gate errors introduced by the last correction. Occasionally, however, single-qubit gates are applied to many, even all of the $K$ logical bits, so we will allow for the errors caused by one transversal operation on $b$, representing one computational step, every time we apply error correction to $b$. That is not to say that on average we correct the whole computer once for each step in the computation. Rather, we can fit in many computational steps, as long as they act on different blocks, between corrections. Let $\eta$ be the average number of computational steps per correction of the whole computer. Since there are more than $n w$ time steps needed to prepare the ancillas (eq. (2)), a safe choice is $\eta=w$, where we suppose the computational steps are carried out during the first of the $r$ ancilla preparations. This is consistent with our assumption that operations on different blocks (here, $a$ and $b$ ) can be done in parallel, and it keeps computational and correction operations on $b$ completely seperated in time. The optimal choice for $\eta$ may be considerably larger than $w$, so we are being cautious.

We will first count the bit errors in $b$. We then assume the same number of sign errors arise, and both correctors (bit and sign) must succeed if the computation is not to fail.

The XOR interaction between $b$ and the first ancilla, $a_{x}$, involves $n$ gates for each repetition of the syndrome generation, making $r n$ gate error opportunities, each with probability $\sim(2 / 3) \gamma$ to leave a bit error in $b$. The XOR interaction between $b$ and the second ancilla $a_{z}$ provides a further $r n$ opportunities and also carries bit errors from $a_{z}$ into $b$. The gate errors which escaped detection in the verification of $b$ are mainly those occuring in the last verification (XOR) gate applied to each qubit, and in the $H$ gates, making $2 r n$ opportunities. Note that these error opportunities are uncorrelated with each other and with the other noise in $b$. The single computational step affecting any given block $b$ before correction produces $n$ gate error opportunities. The total number of independent 
gate error opportunities which the $\sigma_{x}$ correction must deal with is therefore

$$
g=n(4 r+1) .
$$

Each ancilla preparation involves $n w+(n+5) / 2-w$ time steps (eq. (2)). A further $2 n+1$ are needed for the xOR gates and measurement of the two ancillas. With $n$ qubits in $b$, this gives $n(n w+(n+5) / 2-w+2 n+1)$ opportunites for storage error in $b$ for each cycle of syndrome generation. In addition, there are approximately $n^{2} / 2$ opportunities for undetected storage errors which are carried from $a_{z}$ into $b$. The total number of storage error opportunities is

$$
s_{s}=n\left((n-1) w+\frac{5}{2} n+\frac{7}{2}\right) r
$$

when the $r$ syndromes are generated in series (fig. 3a), and

$$
s_{p}=n\left((n-1) w+\frac{1}{2} n+\frac{7}{2}+2 n r\right)
$$

when they are generated in parallel (fig. 3b).

The probability that either more bit-errors, or more sign-errors, or both, accumulate in $b$ than can be corrected is

$$
P \simeq 2 \sum_{i=t+1}^{g} \frac{g !}{i !(g-i) !}\left(\frac{2}{3} \gamma+\frac{s}{g} \frac{2}{3} \epsilon\right)^{i}
$$

where $s$ is either $s_{s}$ or $s_{p}$, and we used the fact that for $\epsilon \ll 1$, it is equally likely for something of probability $\epsilon$ to occur $s / g$ different ways as it is for something of probability $\epsilon s / g$ to occur once. Recall that $w=2 t+2$ and $r=t+1$ so $s_{s} / g \simeq n t / 2$.

\section{CODE COMPARISON}

The value of $P$ (eq. (7) ) is plotted in fig. 4 as a function of $\gamma$, for various CSS codes with the required properties. In fig. 4 (a) serial syndrome repetition is assumed, and the case $n \epsilon=\gamma / 2$ is plotted. Fig. 4 (b) shows results using parallel syndrome repetition, taking $n \epsilon=2 \gamma$. It is seen that parallel repetition allows the memory noise to rise by a factor $\sim 4$, for given $P, \gamma$, at the cost of increased storage and parallelism overheads. In all the cases plotted, storage errors dominate $P$. The codes are $[[7,1,3]]$, [[23, 1, 7]] obtained from the famous Golay code [21], and $[[55,1,11]]$ and $[[87,1,15]]$ obtained from selfdual double circulant codes with weights divisible by 4 [22]. Note that slightly more efficient (quadratic residue) CSS codes exist [20].

$P$ is the failure probability per qubit per correction. To achieve a computation of $Q$ steps involving $K$ logical qubits, we require $P<\eta / K Q$. The overhead involved in the ancilla factory method, applied to the block codes we have chosen, is

$$
\begin{aligned}
& \frac{N}{K}=\left\{\begin{array}{ll}
n+2(n+1) & 2 \text { parallel ancillas per block } \\
n+2 r(n+1) & 2 r \text { parallel ancillas per block }
\end{array},\right. \\
& \frac{T}{Q} \simeq((n-1) w+5 n) 2 r K / \eta .
\end{aligned}
$$

The degree of parallelism needed is $3 K$ or $K(1+2 r)$ using serial or parallel syndrome accumulation.

For comparison, a concatenated code based on the 7bit code has overhead

$$
\begin{aligned}
& \frac{N}{K} \simeq\left(\frac{\log \gamma_{0} Q}{\log \gamma_{0} / \gamma}\right)^{\log _{2} 7}, \\
& \frac{T}{Q} \simeq 7^{L-1} 480 K / \eta
\end{aligned}
$$

where the number of levels $L \simeq \log (N / K) / \log 7$. The expression for $N / K$ is from [11], and I estimate $T / Q$ by noting that $(1+1 / 2+1 / 4+\ldots) 7^{L-1} Q / \eta 7$-bit recoveries must be carried per logical qubit, if 2 recoveries are carried out per level. Each correction uses two ancillas as in fig. 1, and the syndrome is generated twice, making $4 \times 60$ elementary operations ${ }^{\text {t. }}$. The degree of parallelism required is approximately $2 K 7^{L-1}$.

To compare methods, let us consider the case suggested by Preskill [11], in order that we can use his estimates for concatenated coding. The example case is that of factorising a 130 digit (430 bit) number using Shor's algorithm. This factorisation problem would take a few months on current classical machines with the best known classical algorithm. Shor's algorithm requires $K \simeq 5 \times 430=2150$ encoded qubits, and of order $10^{9}$ Toffoli gates, using the circuit of Beckman et. al. [23]. Using Shor's fault-tolerant method [3,11], each Toffoli gate requires about 20 fault-tolerant operations such as XOR or state preparation. Therefore we will take $Q=2 \times 10^{10}$, and the ancilla factory with $[[n, 1, d]]$ block coding will succeed if $P<2 \times 10^{-14} \eta$. Tables 1 and 2 show the error rates and overheads needed to achieve this for various CSS codes. For comparison, the more complete analysis reported by Preskill for the the 7-bit code with three levels of concatenation gives $\gamma \simeq \epsilon \simeq 10^{-6}$, with $N \simeq 1.3 \times 10^{6}$ qubits [11]. I estimate $T \sim 10^{17}$ and a required parallelism of order $2 \times 10^{5}$ for this case.

Table 1 gives the results when the $r$ syndromes are extracted in series (eq. (50), and table 2 gives the results when the $r$ syndromes are extracted in parallel (eq. (6)). Examining table 1, we find that the ancilla factory allows the gate error rate with $[[55,1,11]]$ block coding to be an order of magnitude larger than the result for concatenated coding quoted by Preskill, but requires the storage

\footnotetext{
${ }^{4}$ Using Shor's 'cat state' method to evaluate the syndrome, the factor 480 becomes 576 .
} 
error rate to be an order of magnitude smaller. Since gate errors are liable to be more noisy than free evolution in practice, the block code here compares favourably with the concatenated code. Since the block coding method also has a smaller overhead, the conclusion is that it is the best method in this example.

Examining table 2, we find that block coding with parallel syndrome repetition achieves roughly an order of magnitude improvement in the allowed gate error rate, without much change in the store error rate, compared to the concatenated code, with a similar size overhead but smaller time and parallelism. Here again it is the better choice.

\section{DISCUSSION}

It should be underlined that the comparison in tables 1 and 2 only gives a rough indication of the relative merits of concatenated and block coding, because the analysis I have used is approximate, whereas the results quoted in [11] are based on a more careful computer simulation of the flow of errors through the relevant quantum networks. The present results show that a more thorough comparision would be worthwhile. It would be surprising if such an analysis overturned the overall conclusion, however, since that would require the results here presented to be incorrect by about an order of magnitude. Since my result is essentially $P \sim O\left((g \gamma)^{t+1}\right)$ this would require my estimate of $g$ (eq. (4)) to be incorrect by an order of magnitude. However, I have been cautious at various points in the calculation, especially in supposing the syndrome repetition $r$ to be on average as large as $t+1$, so I expect my results to be cautious rather than over-optimisitic. Zalka 24 has suggested accepting the first syndrome, without repetition, whenever it indicates no errors. Such a method would allow $g$ to be reduced, and therefore $\gamma$ to be increased, by about a factor $t$. Also, it would probably be safe to increase $\eta$, the average number of computational steps per correction. Such simple adjustments would probably allow the error rates given in the tables to be improved by about an order of magnitude. Supporting evidence for this statement is obtained by observing that the threshold $\gamma_{0}$ for $7^{L}$-bit concatenated coding in the case of no memory errors $(\epsilon=0)$ is about an order of magnitude larger in Zalka's optimised method 24 than in the corresponding results of Gottesman et. al. 9.11.

Let us now estimate the size of computation for which the $7^{L}$ concatenated code would be preferable to block coding. With concatenation, when we require more computational steps the overheads $N / K$ and $T / Q$ increase, but the required noise level $\gamma, \epsilon$ is roughly unchanged. Let us add one more level of encoding, $L=4$, and compare with the $[[87,1,15]]$ used with parallel ancillas (table 2 ). The noise requirements of the two methods become roughly equivalent if we allow $\gamma$ and $\epsilon$ for the $[[87,1,15]]$ code to be reduced by a factor of 3 from the values in table 2 . The block code then requires 7 times less memory noise, but allows 6 times more gate noise, than the values $10^{-6}$ for the concatenated code. Since the block code requires only a third as many qubits, and much smaller time and parallelism, one may argue that it is still the better choice. In any case, the value of $P$ will fall by $3^{8}=6561$, so the computation size can increase by the same factor. This would permit Shor's algorithm on numbers of 9 times more digits, ie a 1170 digit (3890 bit) number. The important point is not in the details, but simply that block coding is the better choice for all the computation we are likely to contemplate.

Another consideration is the relative ease of applying computational steps fault-tolerantly with the various codes we have considered. The 7-bit code has the advantage of being simple, and so fault-tolerant operations are relatively easy to find. However, after two or three levels of concatenation some of the fault-tolerant operations become quite complex, especially those requiring preparation of a given state (in encoded form). Block coding is advantageous in this respect. The $[[23,1,7]]$ code is obtained from the famous Golay code [21] which is certainly a good choice for fault-tolerant operations because of the many symmetries of the extended Golay code $[24,12,8]$. (for example its weight distribution is $\left.A_{\{0,8,12,16,24\}}=\{1,759,2576,759,1\}\right)$. An interesting possibility is to concatenate the Golay code with the 7bit code. This would produce a similar correction ability to the $7^{3}$ concatenated code, but with half the block size. This may well allow the 'benchmark' of tables 1 and 2 to be improved upon.

An important lesson is that it is not necessary to consider infinite families of codes (such as the Reed Muller codes) when considering the practical possibilities of quantum computation, since the finite set of known codes already contains specimens sufficiently powerful for all the quantum computation we are likely to contemplate.

\section{ANCILLA FACTORY}

Entropy is kept under control in quantum computation by the feeding in of low-entropy prepared states. At the most basic level, one can take these low-entropy prepared states to be states of bare qubits. However, Shor's fault-tolerant method made a significant step forward in focussing attention on the low-entropy preparation of entangled states (the so-called 'cat' states). The ancilla factory pushes this a stage further. One may wonder whether there is more to be gained by taking another step in the same direction. This would involve preparing well-chosen entangled states of larger ancillas, aiming to reduce the number of ways in which errors can be produced in, or coupled into, each block $b$ during correction. However, unless some radically different approach to quantum error correction can be found, there is little 
further to be gained in this direction. The reason is that any corrector must gather sufficient information from $b$ to learn the bit and sign errors in all the $n$ bits of $b$. In order to couple this information out of $b$ into some other system (whether quantum or classical), at least $2 n$ XOR gates must operate on $b$. If on average the number of syndrome repetitions can be $r \simeq 1$ rather than $t+1$, which seems likely, then this minimum is achieved by the ancilla factory for all stabilizer codes [12].

The main possibility for further improvements is in the preparation of the ancilla state. Fig. 5 shows a network to prepare a 7-bit ancilla for the $[[7,1,3]]$ code using fewer gates than fig. 1. The idea is to take advantage of the structure in the generator, in which not all error propagation routes are open, so that the verifier need not carry out all the parity checks in the $[7,3,4]$ check matrix. Similar networks exist for more complicated codes.

In this paper I have supposed that the only way the quantum computer can be manipulated is through quantum network methods, ie preparation and measurement of qubits in the $\{|0\rangle,|1\rangle\}$ basis, and quantum gates. However, other methods may be possible. For example, one could engineer the coupling between each ancilla block $a_{x}$ and its environment in such a way that relaxation would place $a_{x}$ in the desired state $\left|0_{E}\right\rangle+\left|1_{E}\right\rangle$.

Any method to realise a 'factory' which delivered reliable ancillas could be used to stabilize the quantum computer. Suppose we have a factory which generates the required ancilla states rapidly, but imperfectly. In that case one could use a recursive purification method to obtain ancillas of high fidelity, as shown in fig. 6 . This is closely related to the purification method for entangled pairs of qubits which can make quantum cryptography secure [25,26].

The main conclusions of this study are as follows. First, since quantum error correction is essentially a method in which processing is used to fight noise, it is meaningless to specify the noise level which can be tolerated without stating the processing power required. The main parameters of the processing power are the scale-up $N / K$, the slow-down $T / Q$, and the degree of parallelism. It makes sense to distinguish the noise level $\gamma$ per gate from the noise per time step $\epsilon$ for resting qubits, but it is important to include both contributions. Even when $\epsilon$ is a hundred times smaller than $\gamma$, memory errors can still be the dominant source of noise.

In previous work I argued that the ancilla factory method is successful because it reduces the operations on the encoded qubits of the computer to a minimum. Here I have further shown that the ancilla state need only be verified against a small subset of the errors it might have; this also is important to the success of the method.

The analysis of the error rates and overheads shows that the ancilla factory is the best known method to stabilize quantum computation. Furthermore, block coding is better than concatenated coding using $7^{L}$-bit codes. The reason is that the efficiency of block codes more than compensates for the advantage of correcting low-order er- rors more often than high-order ones, which is the chief advantage of concatenated coding. The best method may be a combined one, in which a more advanced block code such as the Golay code is concatenated with a simple one such as the 7-bit code.

In order to use these methods in a real quantum computer, it will be necessary to achieve the correct noise properties, chiefly the property of uncorrelated noise at different times and places. A real computer might use small-scale methods applied to individual physical qubits, combined with quantum coding on a larger scale. The small-scale methods would depend on the physics of the particular system, and their chief aim would be to ensure that the noise left to be corrected through a QECC would have the right properties.

I thank the Royal Society and St Edmund Hall, Oxford, for their support.

[1] A. R. Calderbank and P. W. Shor, Phys. Rev. A 54, 1098 (1996).

[2] A. M. Steane, Proc. Roy. Soc. Lond. A 452, 2551 (1996).

[3] P. W. Shor, "Fault-tolerant quantum computation," in Proc. 37th Symp. on Foundations of Computer Science, to be published.

[4] A. Yu. Kitaev, "Quantum error correction with imperfect gates," preprint.

[5] E. Knill, R. Laflamme, "Concatenated quantum codes," (LANL eprint quant-ph/9608012)

[6] A. Yu. Kitaev, "Quantum computing: algorithms and error correction," Russian Mathematical Surveys, to appear.

[7] E. Knill, R. Laflamme and W. H. Zurek, "Accuracy threshold for quantum computation," (LANL eprint quant-ph/9610011)

[8] D. Aharonov and M. Ben-Or, "Fault-tolerant quantum computation with constant error," (LANL eprint quant$\mathrm{ph} / 9611025)$

[9] D. Gottesman, J. Evslin, S. Kakade and J. Preskill, to be published (1996).

[10] E. Knill, R. Laflamme and W. H. Zurek, "Resilient quantum computation: Error Models and Thresholds," (LANL eprint quant-ph/9702058)

[11] J. Preskill, "Reliable quantum computers," (LANL eprint quant-ph/9705031)

[12] A. M. Steane, Phys. Rev. Lett. 78, 2252 (1997).

[13] D. Gottesman, Phys. Rev. A 54, 1862 (1996).

[14] A. R. Calderbank, E. M. Rains, P. W. Shor and N. J. A. Sloane, Phys. Rev. Lett. 78, 405 (1997).

[15] D. P. DiVincenzo and P. W. Shor, Phys. Rev. Lett. 77, 3260 (1996).

[16] D. Gottesman, "A theory of fault-tolerant quantum computation," (LANL eprint quant-ph/9702029)

[17] E. Knill and R. Laflamme, Phys. Rev. A, to be published. (LANL eprint quant-ph/9604034). 
[18] C. H. Bennett, D. DiVincenzo, J. A. Smolin and W. K. Wooters, Phys. Rev. A, submitted (LANL eprint quant$\mathrm{ph} / 9604024)$.

[19] A. M. Steane, Phys. Rev. Lett. 77, 793 (1996).

[20] A. M. Steane, Phys. Rev. A 54, 4741 (1996).

[21] M. J. E. Golay, Proc. IEEE 37, 657 (1949).

[22] F. J. MacWilliams and N. J. A. Sloane, "The theory of error-correcting codes," (North-Holland, Amsterdam, ninth impression 1996.)

[23] D. Beckman, A. Chari, S. Devabhaktuni and J. Preskill, Phys. Rev. A 54, 1034 (1996).

[24] C. Zalka, "Threshold estimate for fault tolerant quantum computing," (LANL eprint quant-ph/9612028)

[25] D. Deutsch, A. Ekert, R. Jozsa, C. Macchiavello, S. Popescu and A. Sanpera, Phys. Rev. Lett. 77, 2818 (1996).

[26] C. H. Bennett, G. Brassard, S. Popescu, B. Schumacher, J. A. Smolin and W. Wootters, Phys. Rev. Lett. 76, 722 (1996).

FIG. 1. Preparation of the state $\left|0_{E}\right\rangle+\left|1_{E}\right\rangle$ in the ancilla, including verification to elliminate sign errors in the final state (ie bit errors before the $H$ gates). Each horizontal line is a bare qubit. A circle at the left of a line represents preparation of $|0\rangle$. A small box represents measurement in the basis $\{|0\rangle,|1\rangle\}$. If the measurement result is 1 , the whole network is restarted. If it is zero the measured qubit is taken to be prepared in $|0\rangle$.

FIG. 2. A simple equivalence used in the analysis. Each horizontal line is an encoded qubit, ie one block. The network on the left is used in practice; the equivalent network on the right helps to analyse the propagation of errors.

FIG. 3. A complete correction of a single block. Each horizontal line is a logical qubit, ie one block. A circle at the left of a line represents preparation of $\left|0_{E}\right\rangle$ with verification against bit errors (cf fig. 1). The $r$ syndromes are either generated in parallel (a) or in series (b). The case $r=2$ is illustrated.

FIG. 4. Failure probability, per correction, per block, (eq. (7) ) as a function of gate error probability $\gamma$, for various codes. By reducing $r$ (on average) it should be possible to achieve the same $P$ with $\gamma$ increased by a factor $\sim t$ (see text, section $\mathrm{V}$, but a more complete analysis is needed to confirm this. Dashed line: [[7, 1,3]]; full line: [[23, 1, 7]]; dash-dotted: [[55, 1, 11]]; dotted: [[87, 1, 15]]. (a) serial repetition of syndromes $\left(s=s_{s}\right)$, taking $n \epsilon=\gamma / 2$. (b) parallel repetition of syndromes $\left(s=s_{p}\right)$, taking $n \epsilon=2 \gamma$.

FIG. 5. Reduced network for generation and verification of the ancilla. This does the same job as fig. 1 but the verification takes advantage of the structure of the generation network. No single gate failure or memory error can produce undetected bit errors in two qubits.
FIG. 6. Recursive network for purification of ancillas. In this illustration the ancillas are tested for both bit errors and sign errors, and a single level of recursion is shown. Ancilla states which survive the purification can be fed into the left of the network to obtain the next level.

\begin{tabular}{clcccc}
\hline \hline & units & conc. & {$[[23,1,7]]$} & {$[[55,1,11]]$} & {$[[87,1,15]]$} \\
$\gamma$ & $10^{-6}$ & 1 & 2.2 & 9 & 17 \\
$\epsilon$ & $10^{-6}$ & 1 & 0.05 & 0.08 & 0.1 \\
$N$ & $10^{5}$ & 13 & 1.5 & 3.6 & 5.7 \\
$T$ & $10^{16}$ & 10 & 0.3 & 2 & 3.9 \\
parallelism & $10^{4}$ & 20 & 0.6 & 0.6 & 0.6 \\
\hline \hline
\end{tabular}

TABLE I. Error rates and overheads required to perform $Q=2 \times 10^{10}$ computational steps on $K=2150$ encoded qubits. The error rates for the $7^{3}$ bit concatenated code are taken from a more careful analysis in 11. The other rates are obtained from eq. (7), using serial repetition of syndromes (2 parallel ancillas used $r$ times), and putting $n \epsilon=\gamma / 2$ (cf fig. 4a). Optimisation (see section $\mathrm{V}$ ) would enable the results shown to be improved upon.

\begin{tabular}{clcccc}
\hline \hline & units & conc. & {$[[23,1,7]]$} & {$[[55,1,11]$} & {$[[87,1,15]]$} \\
$\gamma$ & $10^{-6}$ & 1 & 1.7 & 8 & 19 \\
$\epsilon$ & $10^{-6}$ bit & 1 & 0.15 & 0.3 & 0.44 \\
$N$ & $10^{5}$ & 13 & 4.6 & 16 & 32 \\
$T$ & $10^{16}$ & 10 & 0.3 & 2 & 3.9 \\
parallelism & $10^{4}$ & 20 & 1.9 & 2.8 & 3.7 \\
\hline \hline
\end{tabular}

TABLE II. As table 1, but the results for block coding assume each syndrome is extracted via $2 r$ ancillas used in parallel, and we take $n \epsilon=2 \gamma$, (cf fig. $4 \mathrm{~b}$ ). 


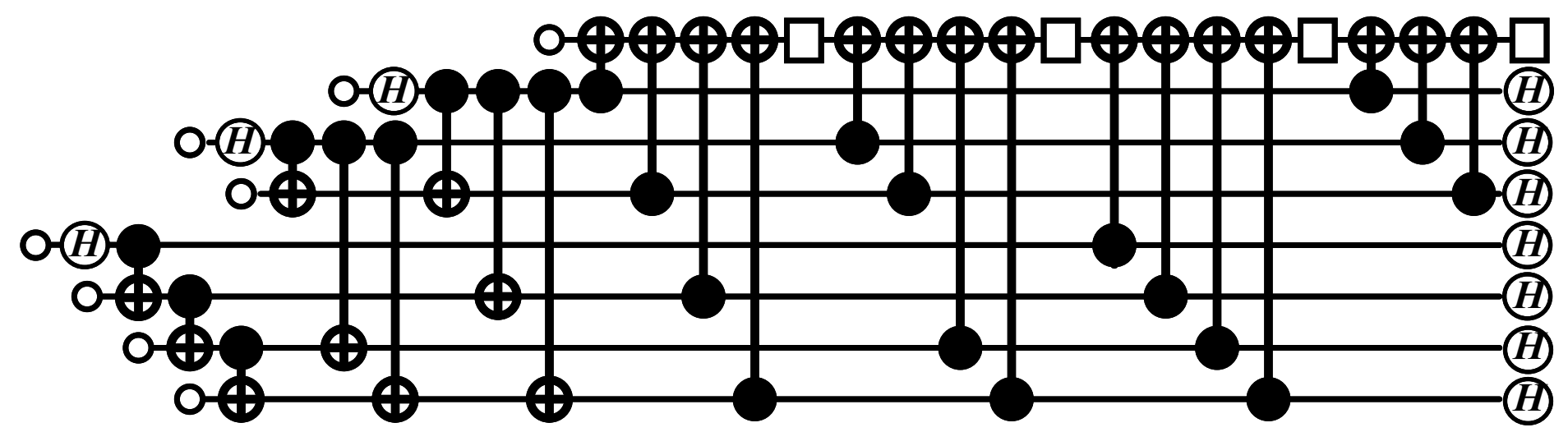




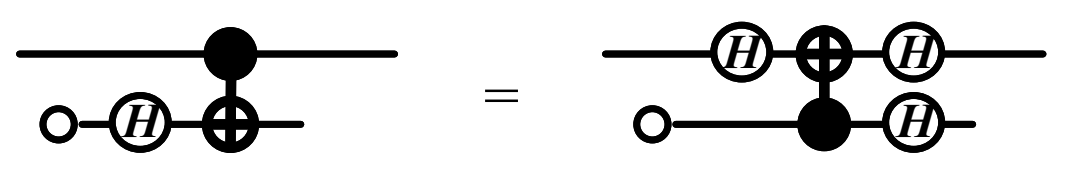


a)

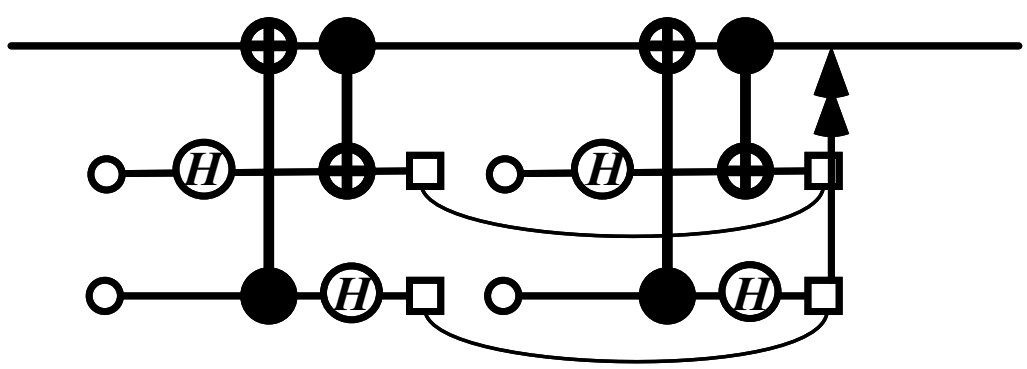

b)

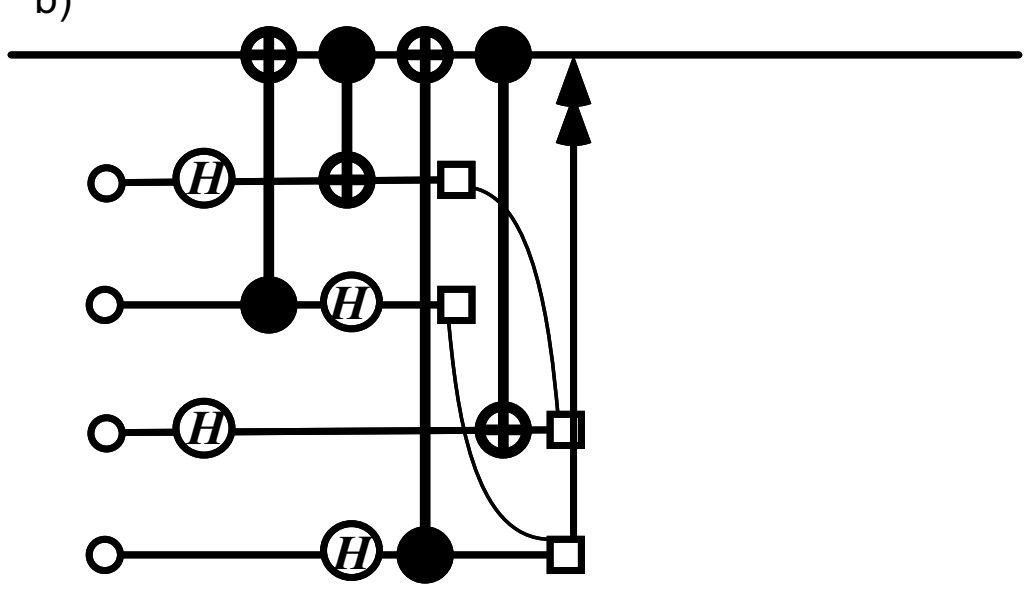




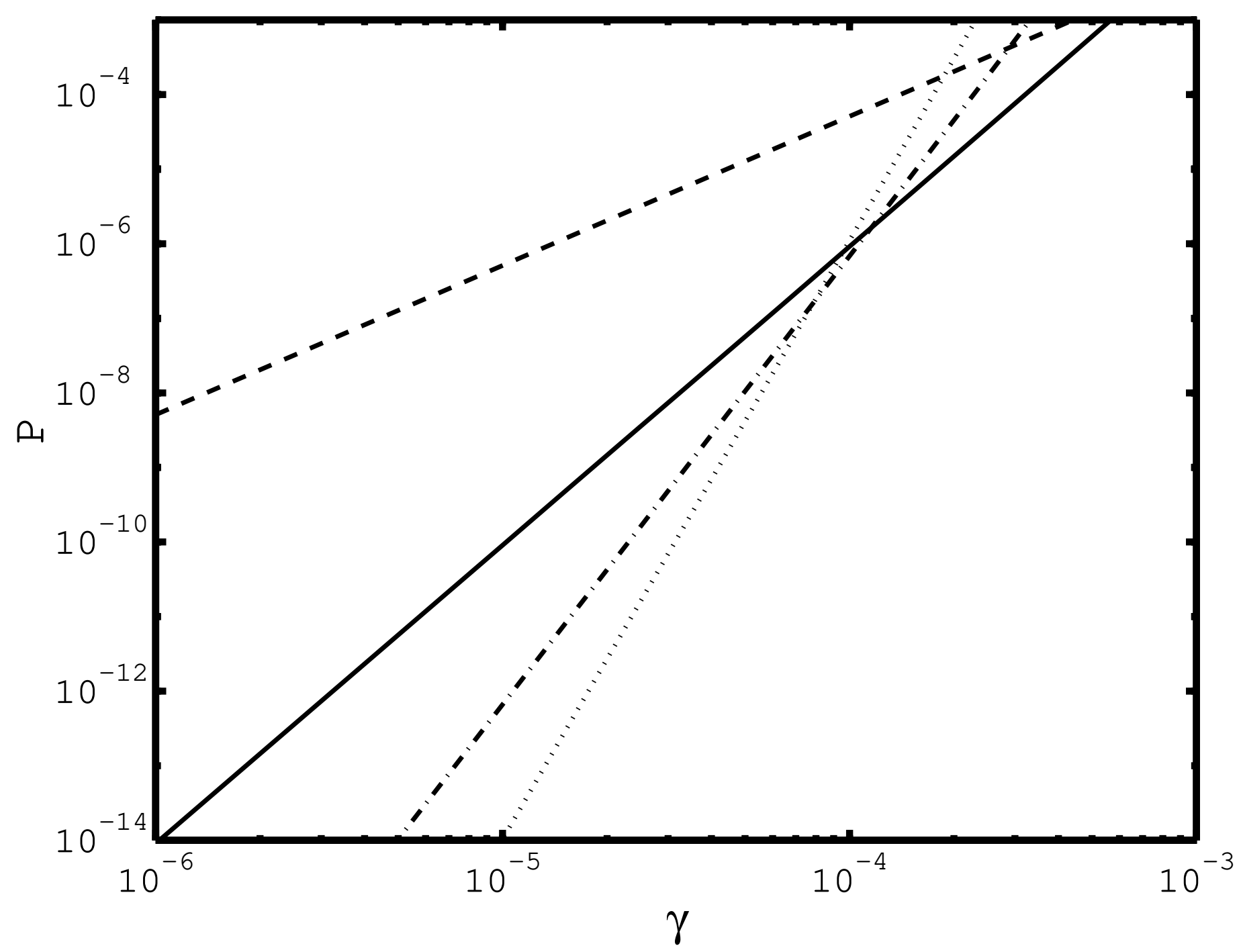




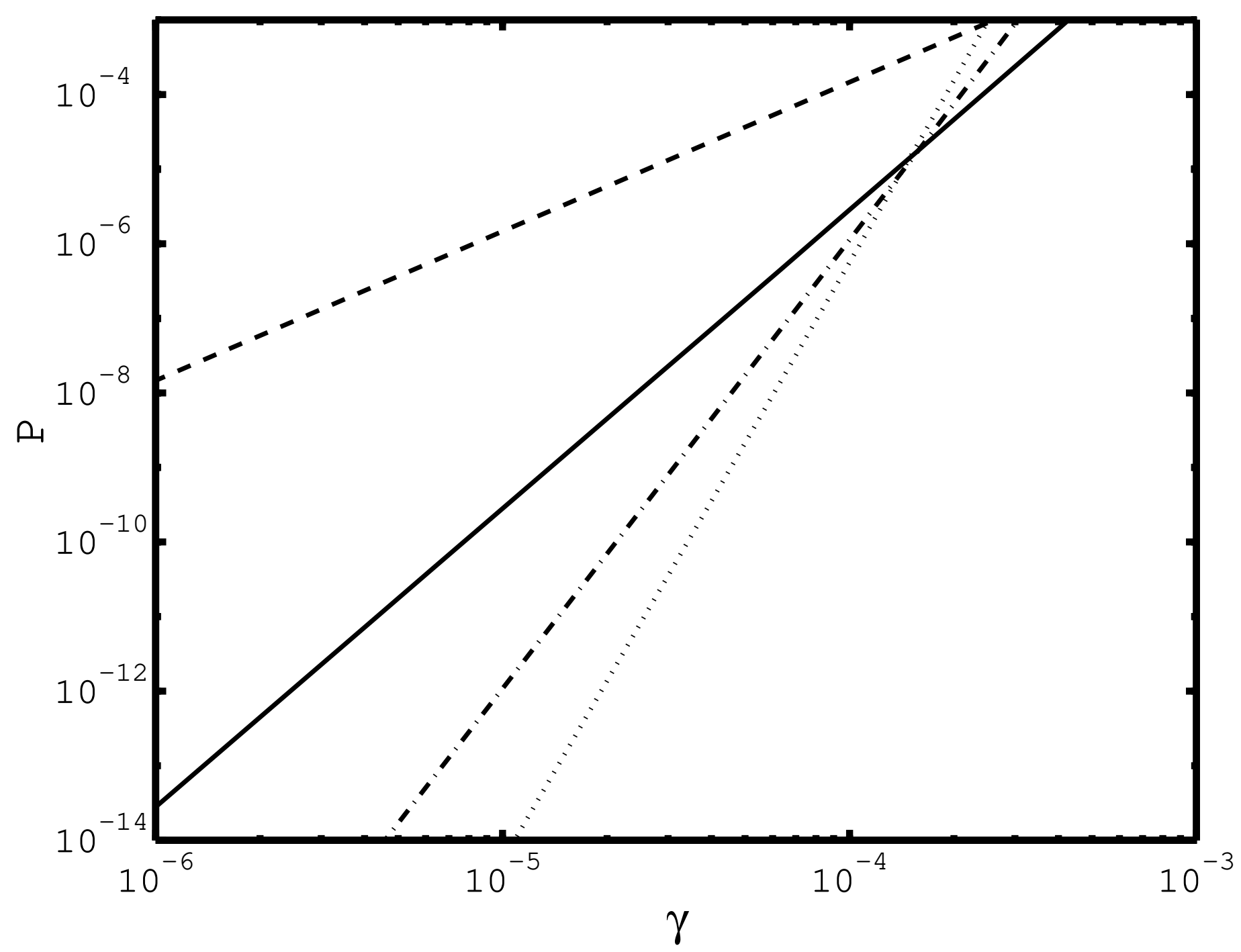




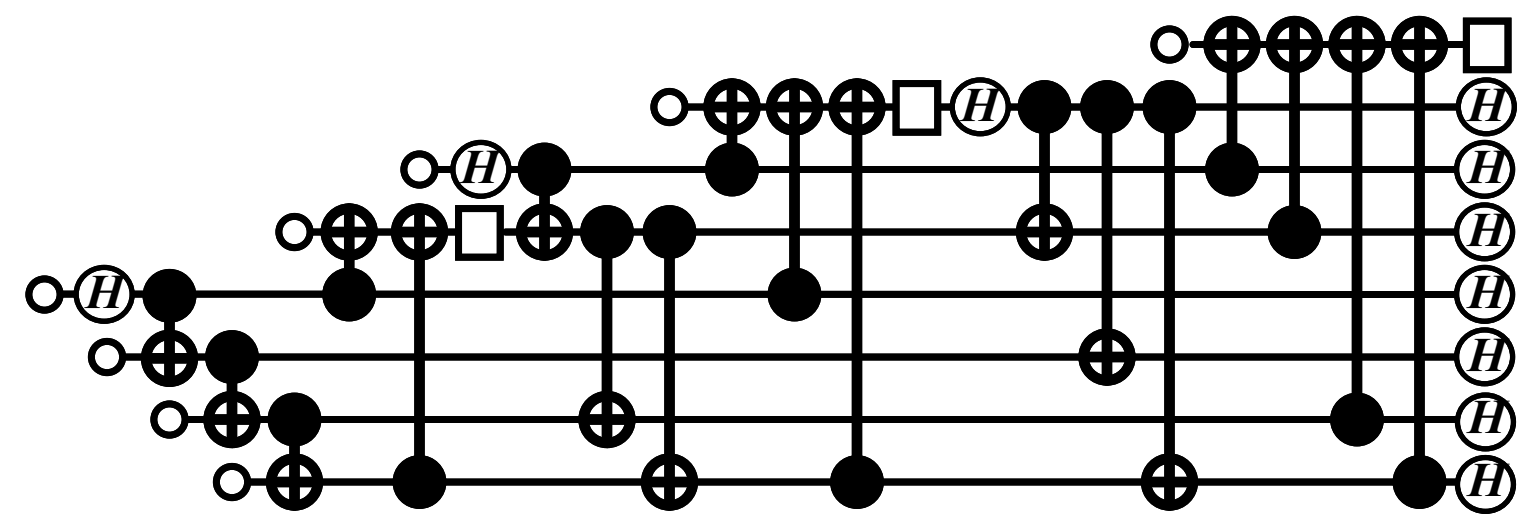




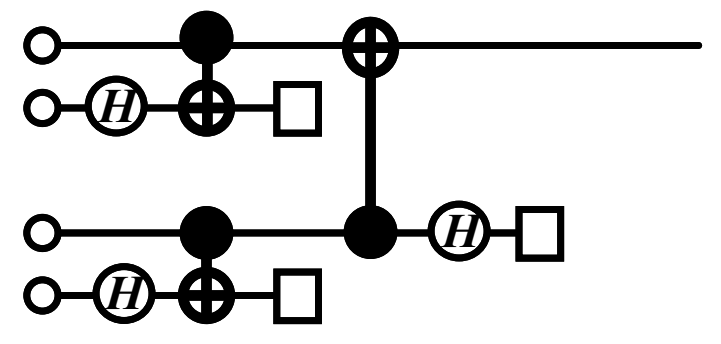

